\title{
ON CYLINDRICITY OF SUBMANIFOLDS OF NONNEGATIVE RICCI CURVATURE IN A MINKOWSKI SPACE
}

\author{
A. BORISENKO AND Y. NIKOLAYEVSKY
}

\begin{abstract}
We consider Finsler submanifolds $M^{n}$ of nonnegative Ricci curvature in a Minkowski space $\mathbb{M}^{n+p}$ which contain a line or whose relative nullity index is positive. For hypersurfaces, submanifolds of codimension two or of dimension two, we prove that the submanifold is a cylinder, under a certain condition on the inertia of the pencil of the second fundamental forms. We give an example of a surface of positive flag curvature in a three-dimensional Minkowski space which is not locally convex.
\end{abstract}

\section{INTRODUCTION}

By Cohn-Vossen [11], a complete two-dimensional manifold of nonnegative Gauss curvature which contains a line (a complete geodesic every arc of which minimises the distance between its endpoints) is flat. Toponogov [19] generalised this result to higher dimension; he proved that if a complete Riemannian manifold $M^{n}$ of nonnegative sectional curvature admits $k$ independent lines, then $M^{n}$ is the Riemannian product $M^{n-k} \times \mathbb{E}^{k}$, where $\mathbb{E}^{k}$ is the Euclidean space of dimension $k$. By the Splitting Theorem of Cheeger-Gromoll, the same is true in the case of nonnegative Ricci curvature [8]. In pseudo-Riemannian settings, the Splitting Theorem was established by Eschenburg [12] and Galloway [13]. Recently Ohta [18] established the differentiable (resp. the isometric) Splitting Theorem for Finsler (resp. Berwald) manifolds of nonnegative weighted Ricci curvature.

In submanifold geometry, one has the following result proved by the first author. Suppose $M^{n}$ is a complete, regular submanifold of a Euclidean space. If the index of relative nullity at every point of $M^{n}$ is bounded below by $k \geq 1$ and if the Ricci curvature of $M^{n}$ is nonnegative, then $M^{n}$ is a cylinder with a $k$-dimensional generatrix [5, Theorem 3.3.2]; a similar result assuming nonnegativity of the sectional curvature has been earlier established by Hartman [15]. In the Finsler settings, similar results were obtained by the first author for hypersurfaces of a Randers space. He proved that a complete hypersurface of nonnegative Ricci curvature of a Randers space which contains a line is a cylinder [6, Theorem 3]. If the latter assumption is replaced by the condition that the index of relative nullity at every point of $M^{n}$ is greater than or equal to $k \geq 1$, then $M^{n}$ is a cylinder with a $k$-dimensional generatrix [6, Theorem 4$]$.

In this paper, we study complete Finsler submanifolds of nonnegative Ricci curvature in an arbitrary Minkowski space. It should be noted that the connection between the sign of the Ricci (or of the flag) curvature of a submanifold in a Minkowski space and the

2010 Mathematics Subject Classification. 53C40, 53C60, 53C21.

Key words and phrases. Finsler submanifold, Ricci curvature, cylindricity, nullity index, flag curvature. The authors were supported by ARC Discovery Grant DP130103485 and by La Trobe University DRP. 
shape of the submanifold is much weaker and is much less understood compared to that for a submanifold of a Euclidean space (see e.g. [7]).

A Minkowski space $\mathbb{M}^{n}$ is a pair $\left(\mathbb{R}^{n}, F\right)$, where $F: \mathbb{R}^{n} \rightarrow[0, \infty)$ is a continuous function (Minkowski norm) satisfying the conditions

(1) $F \in C^{\infty}\left(\mathbb{R}^{n} \backslash\{0\}\right)$;

(2) $F$ is positive homogeneous, that is, $F(\lambda y)=\lambda F(y)$, for all $\lambda>0$ and $y \in \mathbb{R}^{n}$;

(3) The matrix

$$
g_{i j}=\frac{1}{2} \frac{\partial^{2} F^{2}}{\partial y^{i} \partial y^{j}}
$$

where $y^{i}$ are Cartesian coordinates on $\mathbb{R}^{n}$, is positive definite outside of the origin of $\mathbb{R}^{n}$.

A smooth Finsler metric on a smooth manifold is obtained by assigning a Minkowski metric smoothly depending on a point to every tangent space. Given a regular submanifold $M^{n}$ in a Minkowski space $\mathbb{M}^{n+p}=\left(\mathbb{R}^{n+p}, F\right)$, the Minkowski norm $F$ induces a Finsler metric on $M^{n}$. We say that $M^{n}$ is complete if it is (forward and backward) complete for the induced Finsler metric. Clearly, completeness does not depend on the choice of the Minkowski norm $F$ for $\mathbb{R}^{n+p}$ (in particular, one can choose a Euclidean norm).

A submanifold $M^{n}$ in a Minkowski space $\mathbb{M}^{n+p}$ is called a cylinder with a $k$-dimensional generatrix, $k \geq 1$, if $M^{n}$ is the union of $k$-dimensional affine subspaces of $\mathbb{M}^{n+p}$ parallel to a fixed subspace $\mathbb{R}^{k} \subset \mathbb{R}^{n+p}$.

Given a point $P$ on a regular submanifold $M^{n} \subset \mathbb{M}^{n+p}$ we introduce two integer invariants, the index of relative nullity $\mu(P)$ and the type $\mathrm{j}(P)$. Choose an arbitrary Euclidean metric on $\mathbb{R}^{n+p}$ and define the null-space $\mathcal{L}(P) \subset T_{P} M^{n}$ to be the common kernel of all the shape operators of $M^{n}$ at $P$. The index of relative nullity is defined by $\mu(P)=\operatorname{dim} \mathcal{L}(P)$ [9]. The type $\mathrm{j}(P)$ of the point $P$ is defined to be the minimum of the positive index of inertia of the second fundamental forms at $P$ relative to the normals for which the rank of the shape operator is maximal [3]. Note that if $\mathrm{j}(P)=0$, then there exists a normal for which the second fundamental form at $P$ is positive semidefinite. One can easily verify that $\mu(P), \mathrm{j}(P)$ and $\mathcal{L}(P)$ are affine invariants: they do not depend on the choice of the Euclidean metric on $\mathbb{R}^{n+p}$.

We prove the following.

Theorem 1. Let $M^{n}$ be a complete, connected, smooth, regular hypersurface in a Minkowski space $\mathbb{M}^{n+1}$. Suppose that

(a) the Ricci curvature of the induced Finsler metric on $M^{n}$ is nonnegative;

(b) $M^{n}$ contains a straight line of $\mathbb{M}^{n+1}$;

(c) for no $P \in M^{n}$, we have $\mathrm{j}(P)=1$.

Then $M^{n}$ is a cylinder.

An extra condition (like our condition (c)) as compared to the Euclidean case is most likely unavoidable for the claim to hold true - in Section 4 we construct an example of a surface $M^{2} \in \mathbb{M}^{3}$ whose flag curvature is nonnegative, but which is locally saddle (that is, locally $\mathrm{j}=1$ ). Note that in higher codimension, it gets even "worse": by [7, Theorem 1.4], any two-dimensional Finsler metric admits a locally saddle embedding in some $\mathbb{M}^{4}$. In 
contrast, in the Riemannian case, the fact that the Ricci curvature is nonnegative forces the type to be zero [5, Lemma 3.3.1], in any dimension and codimension.

For submanifolds of codimension 2 we prove the following.

Theorem 2. Let $M^{n}$ be a complete, connected, smooth, regular submanifold in a Minkowski space $\mathbb{M}^{n+2}$. Suppose that

(a) the Ricci curvature of the induced Finsler metric on $M^{n}$ is nonnegative;

(b) for all $P \in M^{n}$, we have $\mu(P)=k$, where $k \geq 1$;

(c) for no $P \in M^{n}$, we have $\mathrm{j}(P) \in\{1,2\}$.

Then $M^{n}$ is a cylinder with a $k$-dimensional generatrix.

A submanifold $M^{n}$ in a Minkowski space $\mathbb{M}^{n+p}$ is said to be $k$-ruled, $k \geq 1$, if $M^{n}$ is locally foliated by domains of $k$-dimensional affine subspaces of $\mathbb{M}^{n+p}$. We have the following local result.

Theorem 3. Let $M^{n}$ be a smooth, regular submanifold in a Minkowski space $\mathbb{M}^{n+p}$. Suppose $M^{n}$ is k-ruled. If the Ricci curvature of the induced Finsler metric on $M^{n}$ is nonnegative, then $\mu(P) \geq k$, for all $P \in M^{n}$.

For surfaces, this implies the following global fact.

Theorem 4. Suppose $M^{2}$ is a complete, connected, smooth, regular, ruled surface in a Minkowski space $\mathbb{M}^{2+p}$. If the flag curvature of the induced Finsler metric on $M^{2}$ is nonnegative, then $M^{2}$ is a cylinder.

Throughout the paper, "regular submanifold" means an immersed submanifold. The differentiability conditions can be relaxed from $C^{\infty}$ to $C^{4}$ for the Minkowski norm and to $C^{3}$ for the submanifold.

\section{PRELIMINARIES}

Let $\mathbb{M}^{n+p}=\left(\mathbb{R}^{n+p}, F\right), n \geq 2, p \geq 1$, be a Minkowski space with the Minkowski function $F=F\left(y^{1}, \ldots, y^{n+p}\right)$, where $y^{a}, a=1, \ldots, n+p$, are Cartesian coordinates on $\mathbb{R}^{n+p}$. Denote $H=\frac{1}{2} F^{2}$. The function $H$ is positively homogeneous of degree 2 , smooth outside of the origin, and satisfies $\operatorname{Hess}(H)>0$ outside of the origin.

We adopt the following index convention: $i, j, k, l, r, s=1, \ldots, n ; \alpha, \beta=n+1, \ldots, n+p$; $a, b=1, \ldots, n+p$. In all summations below, the indices run over the corresponding ranges.

Let $M^{n} \subset \mathbb{M}^{n+p}$ be a regular submanifold and let $P \in M^{n}$. We choose the Cartesian coordinates $y^{a}$ in such a way that $P$ is the origin of $\mathbb{R}^{n+p}$ and that $M^{n}$ is locally defined by

$$
y^{\alpha}=f^{\alpha}\left(x^{1}, \ldots, x^{n}\right), \quad y^{i}=x^{i},
$$

with $f^{\alpha}$ smooth functions satisfying $f^{\alpha}(0)=f_{i}^{\alpha}(0)=0$, where here and below the function name with a subscript denotes the partial derivative.

The induced Finsler metric on the submanifold $M^{n}$ is given by

$$
S(x, u)=F\left(u^{1}, \ldots, u^{n}, u^{i} f_{i}^{1}, \ldots, u^{i} f_{i}^{p}\right),
$$

where here and below we adopt the Einstein summation convention. 
The fundamental tensor on $M^{n}$ is given by

$$
g_{i j}=\frac{\partial^{2}}{\partial u^{i} \partial u^{j}}\left(\frac{1}{2} S^{2}\right)=H_{i j}+H_{i \alpha} f_{j}^{\alpha}+H_{j \alpha} f_{i}^{\alpha}+H_{\alpha \beta} f_{i}^{\alpha} f_{j}^{\beta},
$$

where the partial derivatives of $H$ are evaluated at the points of $M^{n}$ [10].

In particular, at the origin $x=0$ we have

$$
g_{i j}(0, u)=H_{i j}(0, u) .
$$

The spray coefficients of $M^{n}$ are given by

$$
G^{i}=\frac{1}{4} g^{i j}\left(u^{k} \frac{\partial^{2}}{\partial x^{k} \partial u^{j}}\left(S^{2}\right)-\frac{\partial}{\partial x^{j}}\left(S^{2}\right)\right)=\frac{1}{2} g^{i j} f_{k l}^{\alpha} u^{k} u^{l}\left(H_{j \alpha}+H_{\alpha \beta} f_{j}^{\beta}\right),
$$

and so at the origin $x=0$ we have

$$
G^{i}(0, u)=\frac{1}{2} h_{\alpha}^{i} f_{k l}^{\alpha} u^{k} u^{l}=\frac{1}{2} h_{\alpha}^{i} \kappa^{\alpha}
$$

where we denote

$$
h_{\alpha}^{i}(u)=g^{i j}(0, u) H_{j \alpha}(0, u) \quad \text { and } \quad \kappa^{\alpha}(u)=f_{k l}^{\alpha} u^{k} u^{l} .
$$

We then compute the Ricci tensor

$$
R_{k}^{i}(u)=2 \frac{\partial G^{i}}{\partial x^{k}}-u^{j} \frac{\partial^{2} G^{i}}{\partial x^{j} \partial u^{k}}+2 G^{j} \frac{\partial^{2} G^{i}}{\partial u^{j} \partial u^{k}}-\frac{\partial G^{i}}{\partial u^{j}} \frac{\partial G^{j}}{\partial u^{k}}
$$

at the origin $x=0$. A straightforward but somewhat tedious calculation gives

$$
\begin{aligned}
R_{k}^{i}(u)= & -\frac{1}{2} \frac{\partial h_{\alpha}^{i}}{\partial u^{k}} f_{j l s}^{\alpha} u^{j} u^{l} u^{s} \\
& -\left(\frac{3}{4} \frac{\partial h_{\alpha}^{i}}{\partial u^{j}} \frac{\partial h_{\beta}^{j}}{\partial u^{k}}+\frac{1}{2} \frac{\partial}{\partial u^{k}}\left(g^{i j} \frac{\partial}{\partial u^{j}}\left(H_{\alpha \beta}-h_{\alpha}^{s} H_{s \beta}\right)\right) \kappa^{\alpha} \kappa^{\beta}\right. \\
& +\left(\frac{3}{2} \frac{\partial h_{\alpha}^{i}}{\partial u^{k}} h_{\beta}^{j}-\frac{1}{2} \frac{\partial}{\partial u^{k}}\left(g^{i j}\left(H_{\alpha \beta}-h_{\beta}^{s} H_{s \alpha}\right)\right)\right) \kappa^{\beta} f_{j l}^{\alpha} u^{l} \\
& -\frac{1}{2} g^{i j}\left(\frac{\partial}{\partial u^{j}}\left(H_{\alpha \beta}-h_{\alpha}^{s} H_{s \beta}\right)\right) \kappa^{\beta} f_{k l}^{\alpha} u^{l} \\
& +g^{i l}\left(H_{\alpha \beta}-h_{\alpha}^{s} H_{s \beta}\right)\left(f_{k l}^{\beta} f_{j r}^{\alpha}-f_{j l}^{\beta} f_{k r}^{\alpha}\right) u^{j} u^{r},
\end{aligned}
$$

where all the partial derivatives of $H$ are evaluated at the point $(u, 0)$ and all the partial derivatives of $f$, at the point $x=0$. It follows that the Ricci curvature of the induced Finsler metric at the origin in the direction $u$ is given by

$$
\operatorname{Ric}(u)=R_{i}^{i}(u)=\xi_{\alpha} f_{j l s}^{\alpha} u^{j} u^{l} u^{s}+\zeta_{\alpha \beta} g^{i l}\left(f_{i l}^{\beta} f_{j r}^{\alpha}-f_{j l}^{\beta} f_{i r}^{\alpha}\right) u^{j} u^{r}-\eta_{\alpha \beta} \kappa^{\alpha} \kappa^{\beta}-\rho_{\alpha \beta}^{i} \kappa^{\beta} f_{i l}^{\alpha} u^{l},
$$

where

$$
\begin{array}{rlrl}
\xi_{\alpha} & =-\frac{1}{2} \frac{\partial h_{\alpha}^{i}}{\partial u^{i}}, & \zeta_{\alpha \beta} & =H_{\alpha \beta}-h_{\alpha}^{s} H_{s \beta}, \\
\eta_{\alpha \beta}=\frac{3}{4} \frac{\partial h_{\alpha}^{i}}{\partial u^{j}} \frac{\partial h_{\beta}^{j}}{\partial u^{i}}+\frac{1}{2} \frac{\partial}{\partial u^{i}}\left(g^{i j} \frac{\partial \zeta_{\alpha \beta}}{\partial u^{j}}\right), & \rho_{\alpha \beta}^{i}=3 \xi_{\alpha} h_{\beta}^{i}+\frac{1}{2} \frac{\partial}{\partial u^{j}}\left(g^{i j} \zeta_{\alpha \beta}\right)+\frac{1}{2} g^{i j} \frac{\partial \zeta_{\alpha \beta}}{\partial u^{j}} .
\end{array}
$$

Later in the proofs we will use the following simple fact. 
Lemma 1. The $p \times p$ matrix $\zeta_{\alpha \beta}$ is positive definite.

Proof. From (6) and (3) we have $\zeta_{\alpha \beta}=H_{\alpha \beta}-h_{\alpha}^{s} H_{s \beta}=H_{\alpha \beta}-g^{s r} H_{r \alpha} H_{s \beta}$. By specifying the coordinates $y^{\alpha}$ on $\mathbb{R}^{n+p}$ it suffices to prove that $H_{n+1, n+1}-g^{r s} H_{r, n+1} H_{s, n+1}>0$. But from the cofactor decomposition, the latter expression equals det $H[n+1] / \operatorname{det} H[n]$, where $H[m]$ denotes the top left $m \times m$ submatrix of $\operatorname{Hess}(H)$.

\section{Proofs of the TheOREMS}

Proof of Theorem 1. We start with the following local fact.

Proposition 1. Let $M^{n}$ be a regular hypersurface in a Minkowski space $\mathbb{M}^{n+1}$ and let $P \in M^{n}$. Suppose that the Ricci curvature of the induced Finsler metric on $M^{n}$ is nonnegative at $P$ and that $\mathrm{j}(P) \neq 1$. Then for any choice of a Euclidean metric on $\mathbb{M}^{n+1}$, the (Riemannian) second fundamental form of $M^{n}$ at $P$ is semidefinite and the (Ricci and sectional) curvature of the induced Riemannian metric on $M^{n}$ is nonnegative at $P$.

Proof. Choose the Cartesian coordinates $y^{1}, \ldots, y^{n}, y^{n+1}$ on $\mathbb{M}^{n+1}$ as in Section 2 and introduce the Euclidean metric on $\mathbb{R}^{n+1}$ in such a way that they are orthonormal. From (5) (dropping the indices $\alpha$ and $\beta$, as $p=1$ ), the Ricci curvature in the direction $u=\left(u^{i}\right)$ at $P$ is given by

$$
\operatorname{Ric}(u)=\xi(u) f_{j l s} u^{j} u^{l} u^{s}+\kappa(u) \phi(u)-\zeta(u) g^{i l}(u) f_{j l} f_{i r} u^{j} u^{r},
$$

where $\phi(u)=-\eta(u) \kappa(u)-\rho^{i}(u) f_{i l} u^{l}+\zeta(u) g^{i l} f_{i l}$. Note that the matrix of the second fundamental form of $M^{n}$ at $P$ relative to the unit normal $\nu=(0, \ldots, 0,1)$ is given by $\left(f_{i j}\right)$ and that $\kappa(u)=f_{i j} u^{i} u^{j}$ is the normal curvature of $M^{n}$ at $P$ in the direction of $u$. Let $C$ be the isotropic cone in the orthogonal complement to the null-space $\mathcal{L}(P)$, that is, $C=\{u \mid \kappa(u)=0, u \perp \mathcal{L}(P)\}$ and let $C_{*}=C \backslash\{0\}$.

Suppose that $\mathrm{j}(P)>1$. Then the set $C_{*}$ is nonempty and connected, and so on a curve $\gamma \subset C_{*}$ which joins an arbitrary $v \in C_{*}$ to $-v \in C_{*}$, there exists a point $u$ such that $f_{j l s} u^{j} u^{l} u^{s}=0$. From (7) it follows that $\zeta(u) g^{i l}(u) f_{j l} f_{i r} u^{j} u^{r} \leq 0$. But $\zeta(u)>0$ by Lemma 1. It follows that $f_{j l} u^{j}=0$, so that $u \in \mathcal{L}(P)$, a contradiction.

Therefore $\mathrm{j}(P)=0$, and so the second fundamental form at the point $P$ is semidefinite. This property does not depend on the choice of the Euclidean metric on $\mathbb{R}^{n+1}$ and moreover, by the Gauss equation, implies that both the sectional and the Ricci curvature of $M^{n}$ at $P$ are nonnegative.

Returning to the proof of the theorem, choose an arbitrary Euclidean metric on $\mathbb{R}^{n+1}$. As the induced Riemannian metric on the hypersurface $M^{n}$ has nonnegative curvature and as $M^{n}$ contains a line, it is isometric to the Riemannian product $M^{n-1} \times \mathbb{E}^{1}$, with the line being $Q \times \mathbb{E}^{1}$ for some point $Q \in M^{n-1}$ [19]. The proof is now concluded by the following Lemma (note that its hypothesis imposes no restrictions on the curvature).

Lemma ([4], [5, Lemma 3.3.2]). Suppose a complete Riemannian manifold $N^{n}$ is isometric to the metric product $N^{n-k} \times \mathbb{E}^{k}$, and let $\iota: N^{n} \rightarrow \mathbb{E}^{n+p}$ be an isometric $C^{0}$-immersion. If for some $Q \in N^{n-k}$ the image $\iota\left(Q \times \mathbb{E}^{k}\right)$ is a $k$-dimensional affine subspace of $\mathbb{E}^{n+p}$, then $\iota\left(N^{n}\right)$ is a cylinder with a k-dimensional generatrix. 
Proof of Theorem 2. We use the following local fact.

Proposition 2. Let $M^{n}$ be a regular submanifold in a Minkowski space $\mathbb{M}^{n+2}$ and let $P \in M^{n}$. Suppose that the Ricci curvature of the induced Finsler metric on $M^{n}$ is nonnegative at $P$. Then $\mathrm{j}(P) \leq 2$.

The proof of Proposition 2 is based on the following algebraic (or rather topological) lemma.

Lemma 2. Let $\phi_{1}, \phi_{2}$ be two quadratic forms and $\psi_{1}, \psi_{2}$ be two cubic forms on $\mathbb{R}^{n}$. Suppose that the positive index of inertia of any linear combination $\lambda_{1} \phi_{1}+\lambda_{2} \phi_{2}$ of maximal rank is at least 3 . Then the four forms $\phi_{1}, \phi_{2}, \psi_{1}, \psi_{2}$ have a common zero on $\mathbb{R}^{n} \backslash\{0\}$.

Assuming Lemma 2 we give the proof of Proposition 2.

Proof of Proposition 2. Choose Cartesian coordinates $y^{1}, \ldots, y^{n+2}$ on $\mathbb{M}^{n+2}$ as in Section 2 and introduce the Euclidean metric on $\mathbb{R}^{n+2}$ in such a way that they are orthonormal. If $\mathrm{j}(P)>2$, then applying Lemma 2 to the restriction of the quadratic forms $f_{i j}^{\alpha} u_{i} u_{j}$ and the cubic forms $f_{i j l}^{\alpha} u_{i} u_{j} u_{l}, \alpha=n+1, n+2$, to $\mathcal{L}(P)^{\perp}$ we find a nonzero vector $u=\left(u^{i}\right) \in$ $T_{P} M^{n}$ orthogonal to $\mathcal{L}(P)$ and such that $\kappa^{\alpha}(u)\left(=f_{i j}^{\alpha} u_{i} u_{j}\right)=0$ and $f_{i j l}^{\alpha} u_{i} u_{j} u^{l}=0$, for $\alpha=n+1, n+2$. But then from (5), the Ricci curvature in the direction $u=\left(u^{i}\right)$ at $P$ is given by $\operatorname{Ric}(u)=-\zeta_{\alpha \beta} g^{i l} f_{j l}^{\beta} f_{i r}^{\alpha} u^{j} u^{r}$. By assumption, $\operatorname{Ric}(u) \geq 0$, and so by Lemma 1 we have $f_{i r}^{\alpha} u^{r}=0$ at $P$, for $\alpha=n+1, n+2$, and all $i=1, \ldots, n$. But then $u \in \mathcal{L}(P)$, a contradiction.

The claim of the theorem now follows from Proposition 2 and [6, Theorem 2].

Proof of Lemma 2. Denote $C$ the set (the cone) of common zeros of the quadratic forms $\phi_{1}$ and $\phi_{2}$ on $\mathbb{R}^{n}$, and let $C_{*}=C \backslash\{0\}$. We use a version of the Borsuk-Ulam Theorem. Let $\gamma^{\prime}:[0,1] \rightarrow C_{*}$ be a path joining two antipodal points and let $\gamma=\gamma^{\prime} \cup\left(-\gamma^{\prime}\right)$ (that is, $\gamma:[0,2] \rightarrow C_{*}$ is defined by $\gamma(t)=\gamma^{\prime}(t)$ for $t \in[0,1]$ and $\gamma(t)=-\gamma^{\prime}(t-1)$ for $t \in[1,2])$. If the cubic forms $\psi_{1}, \psi_{2}$ have no common zeros on $C_{*}$, we can define the map $\Psi: C_{*} \rightarrow S^{1}$ by $\Psi(u)=\left(\psi_{1}(u), \psi_{2}(u)\right) / \sqrt{\psi_{1}^{2}(u)+\psi_{2}^{2}(u)}$. Note that $\Psi$ maps antipodal points to antipodal points, and so the loop $\Psi(\gamma)$ represents a nontrivial (odd) element in $\mathbb{Z}=\pi_{1}\left(S^{1}\right)$. If we can construct the path $\gamma^{\prime}$ in such a way that $\gamma$ represents an element of a finite order in $\pi_{1}\left(C_{*}\right)$, we get a contradiction (in fact, in the proof below, our $\gamma$ will always be contractible on $C_{*}$, with a single exception).

The topology of $C_{*}$ (more precisely, of its intersection with the unit sphere $S^{n-1}$ ) is completely described when the pencil $\lambda_{1} \phi_{1}+\lambda_{2} \phi_{2}$ is generic [14]. There are three conditions which define a generic pencil. Relative to some Cartesian coordinates on $\mathbb{R}^{n}$, the forms $\phi_{1}, \phi_{2}$ are represented by symmetric matrices $A_{1}, A_{2}$ respectively. The first condition is that $\operatorname{det}\left(\lambda_{1} A_{1}+\lambda_{2} A_{2}\right)$ is not identically zero (and so we can assume that the matrix $A_{2}$ is nonsingular). The second condition is that the matrix $A_{2}^{-1} A_{1}$ is semisimple (is diagonalisable over $\mathbb{C}$ ). The third condition is that $C \cap S^{n-1}$ is a smooth manifold; this is equivalent to the fact that for no $u \in C_{*}$, the vectors $A_{1} u$ and $A_{2} u$ are linear dependent. It is easy to see that by a small perturbation, any pencil can be made generic; however, in our proof we have to be a little more careful not to violate the inertia assumption of the lemma. We call the type of a pencil of quadratic forms (or of the pencil of associated 
symmetric matrices relative to some basis) the minimum of the positive index of inertia of all its elements of the maximal rank. We will first show that any pencil of type at least 3 can be arbitrarily close approximated by a generic pencil of type at least 3 . Then using the result of [14] and the arguments in the first paragraph we will show that for every such generic pencil $\lambda_{1} \phi_{1}^{\prime}+\lambda_{2} \phi_{2}^{\prime}$, the forms $\phi_{1}^{\prime}, \phi_{2}^{\prime}, \psi_{1}$ and $\psi_{2}$ have a common zero on the unit sphere $S^{n-1} \subset \mathbb{R}^{n}$. Then the claim of the lemma follows by compactness.

Without loss of generality we can assume that the common null-space of $\phi_{1}$ and $\phi_{2}$ is trivial (as otherwise we can restrict all four forms $\phi_{1}, \phi_{2}, \psi_{1}, \psi_{2}$ to its orthogonal complement). Furthermore, using the canonical form of a pencil of symmetric matrices given in [17, Theorem 9.2] we obtain that the condition $\operatorname{det}\left(\lambda_{1} A_{1}+\lambda_{2} A_{2}\right) \equiv 0$ implies that $\mathbb{R}^{n}$ splits into the direct sum of subspaces $V$ and $V^{\prime}$ orthogonal with respect to both $\phi_{1}$ and $\phi_{2}$, with $\operatorname{dim}(V)=2 m+1, m \geq 1$, and such that the restriction of $\lambda_{1} A_{1}+\lambda_{2} A_{2}$ to $V$ relative to a particular basis for $V$ is given by $Q\left(\lambda_{1}, \lambda_{2}\right)=\left(\begin{array}{cc}0 \\ T^{t}\left(\lambda_{1}, \lambda_{2}\right)\end{array} \begin{array}{c}T\left(\lambda_{1}, \lambda_{2}\right) \\ 0\end{array}\right)$, where $T\left(\lambda_{1}, \lambda_{2}\right)$ is the $m \times(m+1)$ matrix of the form

$$
T\left(\lambda_{1}, \lambda_{2}\right)=\left(\begin{array}{ccccc}
0 & & \lambda_{1} & \lambda_{2} \\
& . & . & \cdot & \\
& \lambda_{1} & \lambda_{2} & & 0
\end{array}\right) .
$$

Note that the pencil $Q\left(\lambda_{1}, \lambda_{2}\right)$ has an isotropic subspace $V_{0}$ of dimension $m+1$, which is a common isotropic subspace of the forms $\phi_{1}$ and $\phi_{2}$. If $m \geq 2$, then $\operatorname{dim} V_{0} \geq 3$. Suppose $m=1$. As the positive index of inertia of all nonzero elements of the pencil $Q\left(\lambda_{1}, \lambda_{2}\right)$ is 1 , the restriction of the pencil $\lambda_{1} \phi_{1}+\lambda_{2} \phi_{2}$ to $V^{\prime}$ has type at least 2 . Then $\operatorname{dim} V^{\prime} \geq 4$, and so by [17, Theorem 11.5(ii)], the forms $\phi_{1}$ and $\phi_{2}$ have a common zero $u \in V^{\prime} \backslash\{0\}$, and hence a common 3-dimensional isotropic subspace $V_{0} \oplus \mathbb{R} u$. So in all the cases, $\phi_{1}$ and $\phi_{2}$ have a common isotropic subspace of dimension 3. The unit sphere $S^{2}$ in that subspace is a subset of $C_{*}$. The claim of the lemma now follows from the argument in the first paragraph of the proof if we take for $\gamma$ the equator of $S^{2}$.

We can therefore assume that for our pencil, $\operatorname{det}\left(\lambda_{1} A_{1}+\lambda_{2} A_{2}\right)$ does not vanish identically, and so without loss of generality we can assume that $\operatorname{det} A_{2} \neq 0$. We can then consider the real Jordan form of the matrix $A_{2}^{-1} A_{1}$. The Jordan cells determine the decomposition of $\mathbb{R}^{n}$ into the direct sum of subspaces $V_{j}$ which are pairwise orthogonal with respect to both $\phi_{1}$ and $\phi_{2}$, and such that for each subspace $V_{j}$, there is a basis relative to which the restrictions of the forms $\phi_{1}$ and $\phi_{2}$ are represented by a canonical pair of matrices, as given in [17, Theorem 9.2]. These canonical pairs come in two forms, depending on whether the Jordan cell corresponds to a real eigenvalue or to a pair of complex-conjugate eigenvalues of the matrix $A_{2}^{-1} A_{1}$.

For a subspace $V_{j}$ of dimension $m \geq 1$ corresponding to a real eigenvalue $\alpha$, the canonical form is given by the pencil of $m \times m$ symmetric matrices

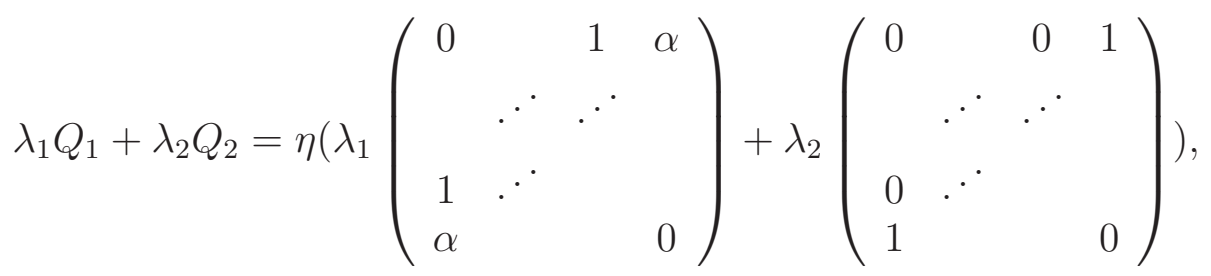


where $\eta= \pm 1$. Now if $m \geq 3$, we replace the top-right and the bottom-left $\alpha$ in $Q_{1}$ by $\alpha+\varepsilon$, with a small $\varepsilon$. It is easy to see that the inertia of the resulting pencil does not change (outside a finite set of points on the circle $\lambda_{1}^{2}+\lambda_{2}^{2}=1$ ), but the matrix $Q_{2}^{-1} Q_{1}$ will now have two different eigenvalues, $\alpha$ and $\alpha+\varepsilon$, so that the pencil $\lambda_{1} Q_{1}+\lambda_{2} Q_{2}$ splits into the direct sum of two smaller pencils of the same form. If $m=2$, we replace the bottom-right zero in $Q_{1}$ by $-\varepsilon$, with a small $\varepsilon>0$. Again, the inertia of the resulting pencil is the same (outside a finite set of points on the circle $\lambda_{1}^{2}+\lambda_{2}^{2}=1$ ), but the resulting matrix $Q_{2}^{-1} Q_{1}$ has two non-real complex-conjugate eigenvalues, $\alpha \pm \mathrm{i} \sqrt{\varepsilon}$. So by an arbitrarily small perturbation without changing the type we can obtain a pencil such that all the subspaces $V_{j}$ corresponding to the real eigenvalues of the matrix $A_{2}^{-1} A_{1}$ are 1-dimensional. The restrictions of the (perturbed) forms $\phi_{1}, \phi_{2}$ to the sum of these subspaces are simultaneously diagonalisable over $\mathbb{R}$.

For a subspace $V_{j}$ of dimension $2 m, m \geq 1$, corresponding to the pair of complexconjugate eigenvalues $\rho \pm \mathrm{i} \nu$ of $A_{2}^{-1} A_{1}$, the canonical form is given by the pencil of $2 \mathrm{~m} \times 2 \mathrm{~m}$ symmetric matrices

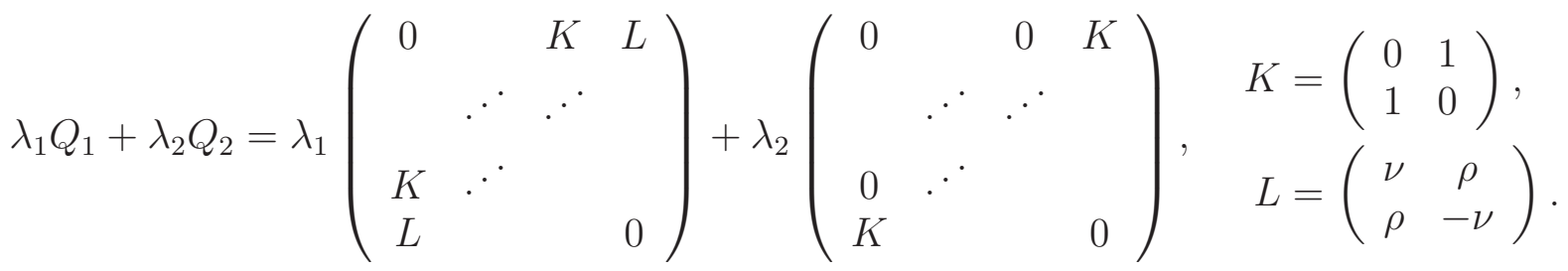

If $m \geq 3$, we replace $\rho$ by $\rho+\varepsilon$ in the top-right and the bottom-left blocks $L$ in $Q_{1}$, with a small $\varepsilon$. This does not change the inertia of the pencil, but the matrix $Q_{2}^{-1} Q_{1}$ for the resulting pencil will have two different pairs of complex-conjugate eigenvalues, $\rho \pm \mathrm{i} \nu$ and $\rho+\varepsilon \pm \mathrm{i} \nu$, and so the pencil $\lambda_{1} Q_{1}+\lambda_{2} Q_{2}$ splits into the direct sum of two smaller pencils of the same form. If $m=2$, we replace the bottom-right $2 \times 2$ zero block in $Q_{1}$ by $\varepsilon I_{2}$, with a small $\varepsilon$. It is not hard to see that the inertia of the resulting pencil is the same, but the resulting matrix $Q_{2}^{-1} Q_{1}$ has two different pairs of complex-conjugate eigenvalues, $\rho \pm \mathrm{i} \sqrt{\nu^{2}+\varepsilon}$ and $\rho \pm \mathrm{i} \sqrt{\nu^{2}-\varepsilon}$. Then the pencil $\lambda_{1} Q_{1}+\lambda_{2} Q_{2}$ splits into the direct sum of two pencils of the same form, with $m=1$.

So by an arbitrarily small perturbation of the given pencil, we can obtain a pencil of the same type such that the matrix $A_{2}^{-1} A_{1}$ is semisimple (over $\mathbb{C}$ ), and we can chose coordinates $\left(u_{i}, v_{t}, w_{t}\right)$ on $\mathbb{R}^{n}$ such that the quadratic forms $\phi_{1}$ and $\phi_{2}$ are given by

$$
\phi_{1}=\sum_{i=1}^{r} \alpha_{i} u_{i}^{2}+\sum_{t=1}^{s}\left(\nu_{t}\left(v_{t}^{2}-w_{t}^{2}\right)+2 \rho_{t} v_{t} w_{t}\right), \quad \phi_{2}=\sum_{i=1}^{r} \beta_{i} u_{i}^{2}+\sum_{t=1}^{s} 2 v_{t} w_{t},
$$

where $r, s \geq 0, r+2 s=n, \beta_{i} \neq 0$ and $\nu_{t}>0$.

Next, we want $C \cap S^{n-1}$ to be a smooth manifold. This is equivalent to the fact that for no $u \in C_{*}$ the vectors $A_{1} u$ and $A_{2} u$ are linear dependent, which is equivalent to the fact that the origin of $\mathbb{R}^{2}$ does not belong to the convex hull of any two vectors $\left(\alpha_{i}, \beta_{i}\right)$ and $\left(\alpha_{j}, \beta_{j}\right), 1 \leq i, j \leq r$. Assuming this condition is violated, we can replace $\phi_{1}$ by $\phi_{1}+2 \varepsilon u_{i} u_{j}$ for some small $\varepsilon$, without changing $\phi_{2}$; it is easy to check that the type of the pencil does not change (but note that $r$ decreases by 2 and $s$ increases by 1 ). Repeating this procedure if necessary we can assume that the quadratic forms $\phi_{1}$ and $\phi_{2}$ are given by 
(8), and for no two $i, j$, the origin of $\mathbb{R}^{2}$ belongs to the convex hull of the vectors $\left(\alpha_{i}, \beta_{i}\right)$ and $\left(\alpha_{j}, \beta_{j}\right)$.

We will now continuously deform the coefficients $\alpha_{i}, \beta_{i}, \nu_{t}, \rho_{t}$ in (8), without violating the above condition on the pairs of vectors $\left(\alpha_{i}, \beta_{i}\right)$ and $\left(\alpha_{j}, \beta_{j}\right)$ and keeping $\nu_{t}$ positive. Note that the inertia of the individual elements of the pencil may change in the process of deformation (for example, we may create or destroy multiple eigenvalues of $A_{2}^{-1} A_{1}$ ), but it is not hard to see that the type of the pencil remains the same. Moreover, the diffeomorphism type of the manifold $C \cap S^{n-1}$ does not change, and we can reduce the forms $\phi_{1}$ and $\phi_{2}$ to the following canonical forms [2, Theorem 1]:

$$
\phi_{1}=\sum_{j=1}^{2 l-1}\left(\cos \frac{2 \pi j}{2 l-1} \sum_{i_{j}=1}^{n_{j}} u_{i_{j}}^{2}\right)+\sum_{t=1}^{s}\left(v_{t}^{2}-w_{t}^{2}\right), \quad \phi_{2}=\sum_{j=1}^{2 l-1}\left(\sin \frac{2 \pi j}{2 l-1} \sum_{i_{j}=1}^{n_{j}} u_{i_{j}}^{2}\right)+\sum_{t=1}^{s} 2 v_{t} w_{t}
$$

where $r=\sum_{j=1}^{2 l-1} n_{j} \geq 0, s \geq 0, r+2 s=n$, and either $l=0$ (we then take $r=0$ ), or $l>0$ and then $n_{j} \geq 1$, for all $j=1, \ldots, 2 l-1$. If $l \geq 2$, we introduce the numbers $d_{j}=n_{j}+\cdots+n_{j+l-2}, j=1, \ldots, 2 l-1$, where the indices are computed modulo $2 l-1$. Then the type of the pencil defined by $\phi_{1}$ and $\phi_{2}$ equals $s+\min \left(d_{j}\right)$ when $l \geq 2$, and equals $s$ when $l \leq 1$. Therefore by our assumption, we have the following inequalities:

$$
s \geq 3, \quad \text { when } l \leq 1 ; \quad s+d_{j} \geq 3, j=1, \ldots, 2 l+1, \quad \text { when } l \geq 2 .
$$

By [14] the submanifold $C \cap S^{n-1}$ is diffeomorphic to

(i) the unit tangent bundle of $S^{s-1}$, if $r=0, s>1$;

(ii) the product $S^{s-1} \times S^{r+s-2}$, if $r>0, l=1, s>0$;

(iii) the product $S^{n_{1}-1} \times S^{n_{2}-1} \times S^{n_{3}-1}$, if $r>0, l=2, s=0$;

(iv) the connected sum $\#_{j=1}^{2 l-1}\left(S^{d_{j}+s-1} \times S^{r-d_{j}+s-2}\right)$, if $r>0, l \geq 2, l+s>2$

(there is also a case $C \cap S^{n-1}=\varnothing$ when $r=0, s \leq 1$, or when $r>0, l=1, s=0$, which may not occur with our assumption on the type). But then from inequalities (10) we can easily see that all the spheres in the above classification are of dimension at least 2, and so $C \cap S^{n-1}$ is always connected and is simply connected in the last three cases, and $\pi_{1}\left(C \cap S^{n-1}\right)=\mathbb{Z}_{2}$ in the first case. This concludes the proof of the lemma.

Note that for the proof, we need much less than the diffeomorphism type of $C \cap S^{n-1}$ : it is sufficient to show that the condition on the type of the pencil implies that $C_{*}$ is connected and that its first Betti number is zero. Instead of the classification theorem of [14] one could have used the results of $[16, \S 2]$; however, we cannot see how to avoid the linear-algebraic "preparation".

In the Riemannian case, the type of a point of a submanifold of an arbitrary codimension in a Euclidean space at which the Ricci curvature is nonnegative is 0 [5, Lemma 3.3.1]. This is no longer true in the Minkowski settings: the possibility $\mathrm{j}(P)=1$ realises already for a two-dimensional surface of positive flag curvature in $\mathbb{M}^{3}$ (as in the example in Section 4). On the other hand, by Proposition 1 and Proposition 2, for $p=1,2$, the type of a point of a submanifold of codimension $p$ of a Minkowski space at which the Ricci curvature is nonnegative is at most $p$. It may be interesting to ask what happens in higher codimension. In that case, constructing the canonical form of a pencil by changing a basis 
is hopeless, but one may use the spectral sequence from [1, Theorem 1] to compute the homology of the common set of zeros of the quadratic forms.

Proof of Theorem 3. Let $P \in M^{n}$. Choose Cartesian coordinates $y^{1}, \ldots, y^{n+p}$ on $\mathbb{M}^{n+p}$ as in Section 2 and introduce the Euclidean metric on $\mathbb{R}^{n+p}$ in such a way that they are orthonormal. In a direction $u=\left(u^{i}\right)$ tangent to a $k$-dimensional generatrix of $M^{n}$ passing through $P$ (there may be more than one such generatrix) we have $\kappa^{\alpha}\left(=f_{i j}^{\alpha} u^{i} u^{j}\right)=0$ and $f_{i j l}^{\alpha} u^{i} u^{j} u^{l}=0$ at $P$, for all $\alpha=n+1, \ldots, n+p$. Then from (5), the Ricci curvature in the direction $u=\left(u^{i}\right)$ at $P$ is given by $\operatorname{Ric}(u)=-\zeta_{\alpha \beta} g^{i l} f_{j l}^{\beta} f_{i r}^{\alpha} u^{j} u^{r}$. By assumption, $\operatorname{Ric}(u) \geq 0$, and so by Lemma 1 we have $f_{i r}^{\alpha} u^{r}=0$ at $P$, for all $\alpha=n+1, \ldots, n+p$ and all $i=1, \ldots, n$. It follows that the tangent space to the $k$-dimensional generatrix passing through $P$ lies in the null-space $\mathcal{L}(P)$.

Proof of Theorem 4. By Theorem 3 we have $\mu(P) \geq 1$, for all $P \in M^{2}$. Choose an arbitrary Euclidean metric on $\mathbb{R}^{2+p}$. Then the induced Riemannian metric on $M^{2}$ is flat and complete, and so the claim follows from [15, Theorem 1.1].

\section{EXAMPLE}

We show that there exists a smooth Minkowski metric on $\mathbb{R}^{3}$, a smooth surface $M^{2} \subset \mathbb{R}^{3}$ and a point $P \in M^{2}$ such that:

- the Gauss curvature of $M^{2}$ at $P$ is negative (relative to any choice of a Euclidean metric on $\mathbb{R}^{3}$ );

- the flag curvature of $M^{2}$ at $P$, with any flagpole, is nonnegative (even positive).

Define the Minkowski function $F=F\left(y^{1}, y^{2}, y^{3}\right)$, where $y^{i}, i=1,2,3$, are Cartesian coordinates on $\mathbb{R}^{3}$ in such a way that $H=\frac{1}{2} F^{2}$ is given by

$$
\begin{aligned}
H\left(y^{1}, y^{2}, y^{3}\right) & =A\left(\left(y^{1}\right)^{2}+\left(y^{2}\right)^{2}\right)+\varepsilon_{1} y^{3} \frac{3 y^{2}\left(y^{1}\right)^{2}-\left(y^{2}\right)^{3}}{\left(y^{1}\right)^{2}+\left(y^{2}\right)^{2}} \\
& +\left(y^{3}\right)^{2}\left(B+\varepsilon_{2} \frac{\left(y^{1}\right)^{6}-15\left(y^{1}\right)^{4}\left(y^{2}\right)^{2}+15\left(y^{1}\right)^{2}\left(y^{2}\right)^{4}-\left(y^{2}\right)^{6}}{\left(\left(y^{1}\right)^{2}+\left(y^{2}\right)^{2}\right)^{3}}\right),
\end{aligned}
$$

where $A$ and $B$ are large positive constants, and $\varepsilon_{1}$ and $\varepsilon_{2}$ are small positive constants; relative to the cylindrical coordinates $r, \theta$, $z$, where $y^{1}=r \cos \theta, y^{2}=r \sin \theta, y^{3}=z$, the function $H$ is given by

$$
H(r, \theta, z)=A r^{2}+\varepsilon_{1} z r \sin (3 \theta)+z^{2}\left(B+\varepsilon_{2} \cos (6 \theta)\right) .
$$

Note that the Minkowski metric so defined is reversible.

Define the surface $M^{2}$ by $y^{3}=f\left(x^{1}, x^{2}\right), y^{1}=x^{1}, y^{2}=x^{2}$, with $f$ a smooth function satisfying $f(0,0)=f_{i}(0,0)=0$ and take $P$ to be the origin $x^{1}=x^{2}=0$.

From (5) and (6), the flag curvature of the surface $M^{2}$ at the point $P$ with the flagpole $u=\left(u^{1}, u^{2}\right)$ is given by

$$
\begin{aligned}
\operatorname{Ric}(u) & =\frac{2 \varepsilon_{1} u^{2}\left(3\left(u^{1}\right)^{2}-\left(u^{2}\right)^{2}\right)}{A\left(\left(u^{1}\right)^{2}+\left(u^{2}\right)^{2}\right)^{2}} f_{i j k} u^{i} u^{j} u^{k}+\frac{P(u)}{4 A^{2}\left(\left(u^{1}\right)^{2}+\left(u^{2}\right)^{2}\right)^{2}}\left(f_{11} f_{22}-f_{12}^{2}\right) \\
& +\frac{Q_{11}(u) f_{11}+Q_{12}(u) f_{12}+Q_{22}(u) f_{22}}{A^{2}\left(\left(u^{1}\right)^{2}+\left(u^{2}\right)^{2}\right)^{4}}\left(f_{11}\left(u^{1}\right)^{2}+2 f_{12} u^{1} u^{2}+(u) f_{22}\left(u^{2}\right)^{2}\right),
\end{aligned}
$$


where

$$
\begin{aligned}
P(u)= & \left(4 A B+4 A \varepsilon_{2}-9 \varepsilon_{1}^{2}\right)\left(u^{1}\right)^{6}+3\left(4 A B-20 A \varepsilon_{2}+15 \varepsilon_{1}^{2}\right)\left(u^{1}\right)^{4}\left(u^{2}\right)^{2} \\
& +3\left(4 A B+20 A \varepsilon_{2}-25 \varepsilon_{1}^{2}\right)\left(u^{1}\right)^{2}\left(u^{2}\right)^{4}+\left(4 A B-4 A \varepsilon_{2}-\varepsilon_{1}^{2}\right)\left(u^{2}\right)^{6}, \\
Q_{11}(u)= & 6\left(u^{1}\right)^{2}\left(\left(3 A \varepsilon_{2}-3 \varepsilon_{1}^{2}\right)\left(u^{1}\right)^{6}+\left(33 \varepsilon_{1}^{2}-51 A \varepsilon_{2}\right)\left(u^{1}\right)^{4}\left(u^{2}\right)^{2}\right. \\
& \left.+\left(65 A \varepsilon_{2}-65 \varepsilon_{1}^{2}\right)\left(u^{1}\right)^{2}\left(u^{2}\right)^{4}+\left(11 \varepsilon_{1}^{2}-9 A \varepsilon_{2}\right)\left(u^{2}\right)^{6}\right), \\
Q_{22}(u)= & 3\left(u^{2}\right)^{2}\left(\left(18 A \varepsilon_{2}-27 \varepsilon_{1}^{2}\right)\left(u^{1}\right)^{6}+\left(115 \varepsilon_{1}^{2}-130 A \varepsilon_{2}\right)\left(u^{1}\right)^{4}\left(u^{2}\right)^{2}\right. \\
& \left.+\left(102 A \varepsilon_{2}-81 \varepsilon_{1}^{2}\right)\left(u^{1}\right)^{2}\left(u^{2}\right)^{4}+\left(\varepsilon_{1}^{2}-6 A \varepsilon_{2}\right)\left(u^{2}\right)^{6}\right), \\
Q_{12}(u)= & 3 u^{1} u^{2}\left(\left(24 A \varepsilon_{2}-33 \varepsilon_{1}^{2}\right)\left(u^{1}\right)^{6}+\left(181 \varepsilon_{1}^{2}-232 A \varepsilon_{2}\right)\left(u^{1}\right)^{4}\left(u^{2}\right)^{2}\right. \\
& \left.+\left(232 A \varepsilon_{2}-211 \varepsilon_{1}^{2}\right)\left(u^{1}\right)^{2}\left(u^{2}\right)^{4}+\left(23 \varepsilon_{1}^{2}-24 A \varepsilon_{2}\right)\left(u^{2}\right)^{6}\right) .
\end{aligned}
$$

We now choose the third derivatives $f_{i j k}$ at zero in such a way that

$$
f_{111}\left(u^{1}\right)^{3}+3 f_{112}\left(u^{1}\right)^{2} u_{2}+3 f_{122} u^{1}\left(u^{2}\right)^{2}+f_{222}\left(u^{2}\right)^{3}=C u^{2}\left(3\left(u^{1}\right)^{2}-\left(u^{2}\right)^{2}\right),
$$

where $C$ is a very large positive number. Then the first term in (12) is large and positive everywhere except for on the three lines $\mathbb{R} u, u=(1,0),(1, \pm \sqrt{3})$, where it is zero. It therefore suffices to choose the second derivatives $f_{i j}$ at zero in such a way that the sum $T(u)$ of the other two terms in (12) is positive at these three points and that $f_{11} f_{22}-f_{12}^{2}<$ 0 . We have

$$
\begin{aligned}
T(1,0) & =\frac{1}{4 A^{2}}\left(a_{1}\left(f_{11} f_{22}-f_{12}^{2}\right)+72 a_{2} f_{11}^{2}\right), \\
T(1, \pm \sqrt{3}) & =\frac{1}{2 A^{2}}\left(2 a_{1}\left(f_{11} f_{22}-f_{12}^{2}\right)+9 a_{2}\left(f_{11}+3 f_{22}-2 \sqrt{3} f_{12}\right)^{2}\right),
\end{aligned}
$$

where $a_{1}=4 A B+4 A \varepsilon_{2}-9 \varepsilon_{1}^{2}, a_{2}=A \varepsilon_{2}-\varepsilon_{1}^{2}$. Take $f_{11}=f_{22}=1, f_{12}=\sqrt{1+\varepsilon_{3}}$, where $\varepsilon_{3}>0$. Then $f_{11} f_{22}-f_{12}^{2}<0$, and for $\varepsilon_{1}$ and $\varepsilon_{3}$ small enough, $T(1,0), T(1, \pm \sqrt{3})>0$.

\section{REFERENCES}

[1] A. A. Agrachëv. The topology of quadratic mappings and Hessians of smooth mappings. In Algebra. Topology. Geometry, Vol. 26 (Russian), Itogi Nauki i Tekhniki, pages 85-124, 162. Akad. Nauk SSSR, Vsesoyuz. Inst. Nauchn. i Tekhn. Inform., Moscow, 1988. Translated in J. Soviet Math. 49 (1990), no. 3, 990-1013.

[2] A. A. Agrachëv and R. V. Gamkrelidze. Quadratic mappings and smooth vector functions: Euler characteristics of level sets. In Current problems in mathematics. Newest results, Vol. 35 (Russian), Itogi Nauki i Tekhniki, pages 179-239. Akad. Nauk SSSR, Vsesoyuz. Inst. Nauchn. i Tekhn. Inform., Moscow, 1989. Translated in J. Soviet Math. 55 (1991), no. 4, 1892-1928.

[3] A. A. Borisenko. The structure of 1-dimensional surfaces with degenerate second quadratic form in n-dimensional Euclidean space. Ukrain. Geometr. Sb., (Vyp. 13):18-27, 2, 1973.

[4] A. A. Borisenko. The structure of a continuous surface that contains a line. Ukrain. Geometr. Sb., (14):21-24, 1973.

[5] A. A. Borisenko. Extrinsic geometry of strongly parabolic multidimensional submanifolds. Uspekhi Mat. Nauk, 52(6(318)):3-52, 1997.

[6] A. A. Borisenko. On the cylindricity of submanifolds containing a line in a Minkowski space. Mat. Sb., 205(7):25-42, 2014.

[7] Dmitri Burago and Sergei Ivanov. On intrinsic geometry of surfaces in normed spaces. Geom. Topol., 15(4):2275-2298, 2011. 
[8] Jeff Cheeger and Detlef Gromoll. The splitting theorem for manifolds of nonnegative Ricci curvature. J. Differential Geometry, 6:119-128, 1971/72.

[9] Shiing-shen Chern and Nicolaas H. Kuiper. Some theorems on the isometric imbedding of compact Riemann manifolds in euclidean space. Ann. of Math. (2), 56:422-430, 1952.

[10] Shiing-Shen Chern and Zhongmin Shen. Riemann-Finsler geometry, volume 6 of Nankai Tracts in Mathematics. World Scientific Publishing Co. Pte. Ltd., Hackensack, NJ, 2005.

[11] Stefan Cohn-Vossen. Totalkrümmung und geodätische Linien auf einfachzusammenhängenden offenen vollständigen Flächenstücken. Rec. Math. Moscou, n. Ser., 1:139-164, 1936.

[12] J.-H. Eschenburg. The splitting theorem for space-times with strong energy condition. J. Differential Geom., 27(3):477-491, 1988.

[13] Gregory J. Galloway. The Lorentzian splitting theorem without the completeness assumption. J. Differential Geom., 29(2):373-387, 1989.

[14] Vinicio Gómez Gutiérrez and Santiago López de Medrano. Topology of the intersections of quadrics II. Bol. Soc. Mat. Mex. (3), 20(2):237-255, 2014.

[15] Philip Hartman. On the isometric immersions in Euclidean space of manifolds with nonnegative sectional curvatures. II. Trans. Amer. Math. Soc., 147:529-540, 1970.

[16] V. A. Krasnov. On the intersection of two real quadrics. Izv. Ross. Akad. Nauk Ser. Mat., 82(1):97$150,2018$.

[17] Peter Lancaster and Leiba Rodman. Canonical forms for Hermitian matrix pairs under strict equivalence and congruence. SIAM Rev., 47(3):407-443, 2005.

[18] Shin-ichi Ohta. Splitting theorems for Finsler manifolds of nonnegative Ricci curvature. J. Reine Angew. Math., 700:155-174, 2015.

[19] V. A. Toponogov. The metric structure of Riemannian spaces of non-negative curvature containing straight lines. Sibirsk. Mat. Ž., 5:1358-1370, 1964.

Department of Differential Equations and Geometry, B.Verkin Institute for Low Temperature Physics and Engineering of the National Academy of Sciences of Ukraine, KHARKIV, 61103, UKRAINE

E-mail address: aborisenk@gmail.com

Department of Mathematics and Statistics, La Trobe University, Melbourne, 3086, Australia

E-mail address: Y.Nikolayevsky@latrobe.edu.au 